\title{
MATHEMATICAL MODEL PREDICTING THE HEAT AND POWER DISSIPATED IN AN ELECTRO- CONDUCTIVE CONTACT IN A HYBRID WOVEN FABRIC
}

\author{
Carla Hertleer ${ }^{1}$, Jeroen Meul ${ }^{1}$, Gilbert De Mey ${ }^{2}$, Simona Vasile ${ }^{3}$, Sheilla A. Odhiambo ${ }^{1,4}$, and Lieva Van Langenhove ${ }^{1}$ \\ 1 Ghent University, Department of Materials, Textiles and Chemical Engineering, Technologiepark 70A, 9052 Zwijnaarde, Belgium +32 92645735 \\ 2 Ghent University, Department of Electronics and Information Systems, Technologiepark 126, 9052 Zwijnaarde, Belgium \\ 3 University College Ghent, Department of Textiles, Fashion and Wood Technologies, Buchtenstraat 11, 9051 Sint-Denijs-Westrem, Belgium \\ 4 Department of Manufacturing, Industrial and Textile Engineering, ACE II PTRE, Moi University, PO Box 3900, Eldoret, Kenya \\ E-mail: sheillatienoodhiambo@gmail.com
}

\begin{abstract}
:
Electro-conductive (EC) yarns can be woven into a hybrid fabric to enable electrical current to flow through the fabric from one component $A$ to another component $B$. These hybrid fabrics form the bases of woven e-textiles. However, at the crossing point of an EC yarn in warp and in weft direction, there is a contact resistance and thus generation of heat may occur in this area. Both phenomena are inseparable: if the contact resistance in the EC contact increases, the generated heat will increase as well. Predicting this electrical and thermal behavior of EC contacts in hybrid woven fabrics with stainless steel yarns is possible with a mathematical model based on the behavior of a metal oxide varistor (MOV). This paper will discuss in detail how this can be achieved.
\end{abstract}

\section{Keywords:}

Hybrid woven fabrics, e-textiles, stainless steel, electro-conductive contacts, contact resistance, metal oxide varistor

\section{Introduction}

Electro-conductive (EC) yarns are basic units of smart textile, as they create a path for current flow or routing of electrical signals [1] in the smart textile system, or sometimes are part of the components of smart textiles. Smart textiles are textiles with additional features such as sensing and/or acting. Heating garments and socks are a few examples of these new emerging smart textiles. Smart textile systems on the other hand are smart textiles with features/components based on electrical circuits. They are also referred to as e-textiles [2]. In an e-textile, electricity is used to power the components comprising the smart textile system. Hence, an electrical circuit has to be composed. Various textile technologies can be applied, such as stitching or embroidering EC yarns between the components [3], but the interconnection can also already be a part of the product, e.g. by weaving or knitting. Making the interconnecting yarns part of the fabric results in a more solid connection with fewer fabrication steps. Figure 1 shows an example of a woven fabric with integrated stainless steel yarns.

Interconnecting the different components by using EC yarns in a hybrid fabric calls for additional attention to the EC contacts. In their paper, Dhawan et al. studied fabric-based electrical circuits with copper wires woven into the fabric [1]. Resistance welding was applied to produce efficient and uniform interconnections in the fabric. The contact resistance of two interlacing stainless steel yarns is already extensively reported in the study by Vasile et al. [4]. The researchers mainly concluded that the diameter of the EC yarn and the weft density had a significant influence on the contact resistance. The properties of knitted EC yarns were studied by Liu et al., and also a resistance model of EC tuck stitches was presented [5].
In this paper, the relationship between the contact resistance and the generated heat in the contact point is studied and is described in a mathematical model based on the behavior of a metal oxide varistor (MOV) [6].

\section{Experiment}

In the event that e.g. a smart phone is used to power the components $\mathrm{C} 1, \mathrm{C} 2$, and $\mathrm{C} 3$ in the electrical circuit of Figures 2 and 3 , it is clear that EC warp and weft yarns of a woven fabric can be used to do the transport of the electrical energy.

The hybrid fabric studied in this paper is composed of two EC yarns and conventional yarns making the base fabric. If a weft and warp EC yarn intersect perpendicular, they make contact,

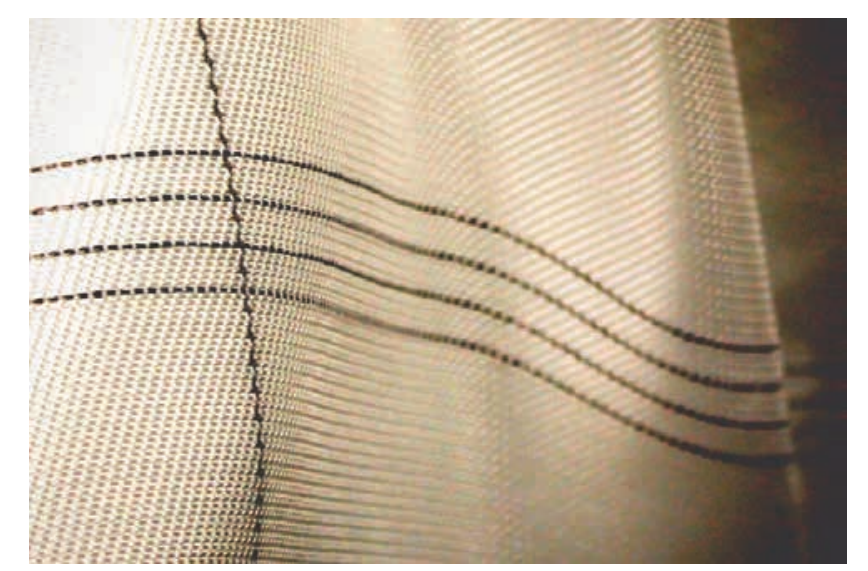

Figure 1. A hybrid woven fabric with stainless steel and polyester yarn in a $3 / 1$ twill pattern. 
an EC contact. A small electric circuit can be created using only a few EC yarns and an EC contact (Figure 3). Two important aspects have to be controlled if a current flows through the EC contact: resistance and heating. In addition, both phenomena are inseparable; if resistance increases, heating increases too. To avoid burns when the EC contact touches the skin, the temperature should be kept below $60^{\circ} \mathrm{C}[7]$.

\section{Materials}

Hybrid fabrics were woven on an airjet loom. They were plain woven fabrics made with $1,100 \mathrm{dtex}$ (130 torsions $/ \mathrm{m}$ ) polyester warp yarns and 1,100 dtex (60 torsions $/ \mathrm{m}$ ) polyester weft yarns as base fabric. The warp density was 18 yarns $/ \mathrm{cm}$, the weft density was 8 yarns $/ \mathrm{cm}$. The base fabric had a specific mass of $309 \mathrm{~g} / \mathrm{m}^{2}$. Four kinds of commercially available stainless steel yarns such as Bekinox VN12.1, VN12.2, VN12.3, and VN12.4 [8] were used to make the hybrid woven fabric. Their characteristics are described in Table 1. The second number in the yarn code is the amount of (VN12.1) bundles that are twisted together to form one yarn and gives an indication of the overall linear mass density of the EC yarns. The yarns were used both as warp and as weft yarn, thus making EC contact points in the hybrid woven fabric. When an EC contact is described, e.g. VN12.4 or VN12.3, the warp yarn is named first and then the weft yarn.

In the experiments, the current was applied by a TTi Power Supply EL $301 \mathrm{R}$, and the voltage was measured using a Fluke $87 \mathrm{~V}$ True RMS Multimeter [9].

The heat generation in the EC contacts was measured using a thermographic camera FLIR T420. The resolution of the camera is $320^{\prime} 240$ pixels with a thermal sensitivity lower than $45 \mathrm{mK}$ at $30^{\circ} \mathrm{C}$.

\section{Methods}

The study of the EC contact point requires examining the overall contact resistance in that point and measuring the generated heat.

First, the EC contact between two stainless steel yarns was studied by hanging two yarns as shown in Figure 4. Three different combinations of yarns were tested as follows: two VN12.1 yarns, two VN12.2 yarns, and two VN12.4 yarns, to represent this kind of yarns. For each combination, three precision weights were attached to the hanging yarns of 2 , 10 , and $100 \mathrm{~g}$. These resemble the contact that warp and weft yarns have in the woven fabric, where a higher weight refers to a higher warp and/or weft density.

Table 1. Electro-conductive yarn characteristics

\begin{tabular}{|c|c|c|c|c|}
\hline & VN12.1 & VN12.2 & VN12.3 & VN12.4 \\
\hline Average thickness $(\mathrm{mm})$ & 295 & 460 & 643 & 775 \\
\hline Linear mass density (tex) & 250 & 500 & 750 & 1,000 \\
\hline Linear resistance $(\mathrm{W} / \mathrm{m})$ & 31.7 & 14.7 & 10.1 & 7.7 \\
\hline
\end{tabular}

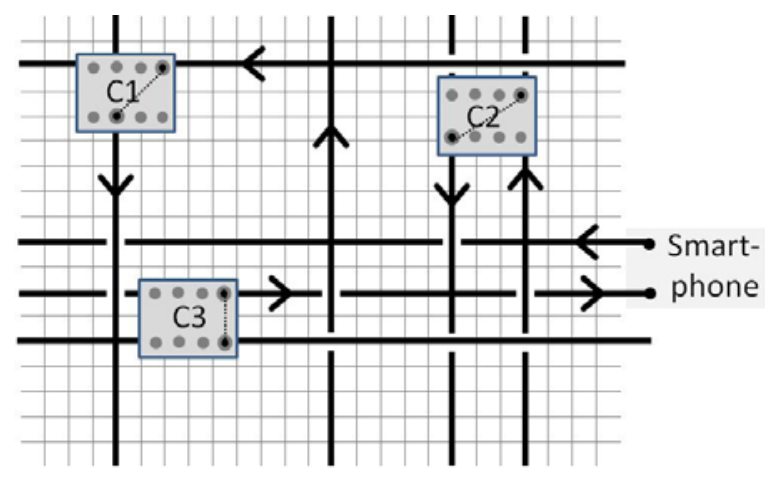

Figure 2. A woven circuit electrically connecting a smart phone to the components $\mathrm{C} 1, \mathrm{C} 2$, and $\mathrm{C} 3$.

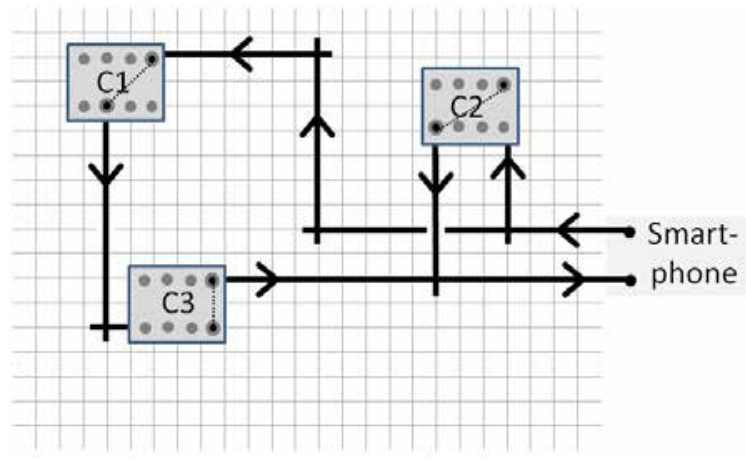

Figure 3. The resulting electro-conductive path.

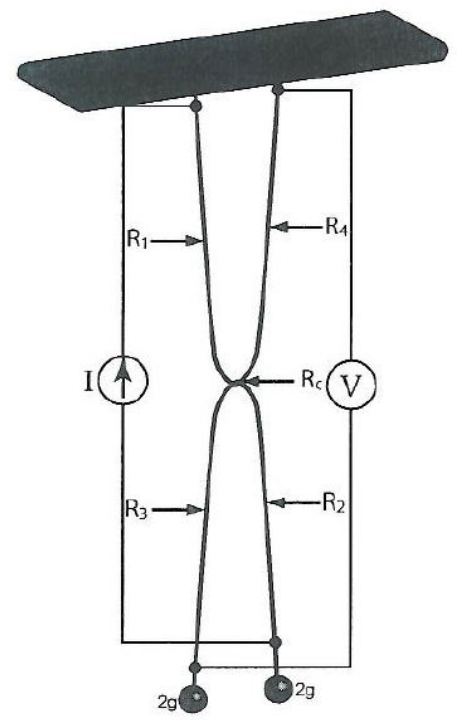

Figure 4. Setup to study the contact between two electro-conductive yarns [10].

To determine the contact resistance with highest accuracy, the four electrode-four contact method was used. A current was introduced in the EC yarns as indicated by $(\mathrm{I})$ in Figures 4 and $5(A)$. The voltage was measured as indicated by $(V)$ in both figures. From the current and the voltage, the contact 
resistance in the contact point was calculated using Ohm's law.

$$
R(\Omega)=\frac{V(V)}{I(A)}
$$

Second, the heat generated in the EC contact point is measured using a thermographic camera, as shown in Figures 5 and 6. All the experiments were done at a point when electrical and thermal stability was reached. This took up to a maximum of five minutes.

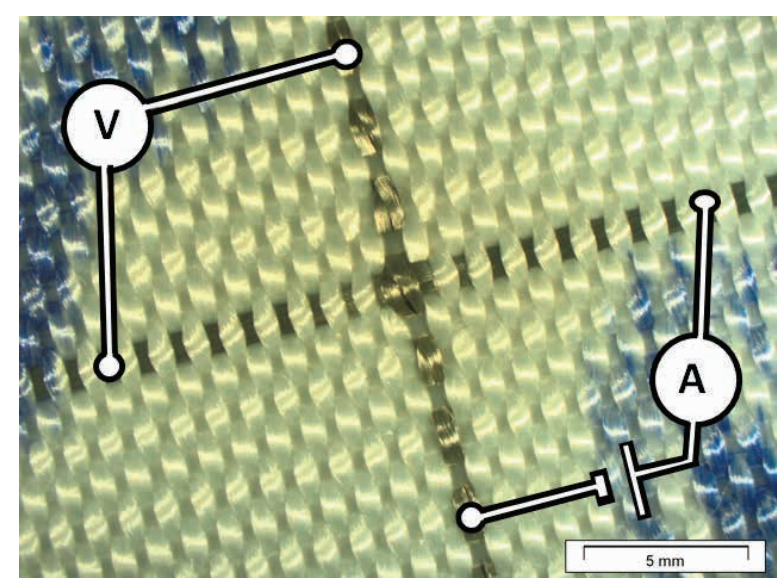

Figure 5. Four electrode-four contact method used to measure voltage (V) as a function of current (A) flowing through the electro-conductive contact.

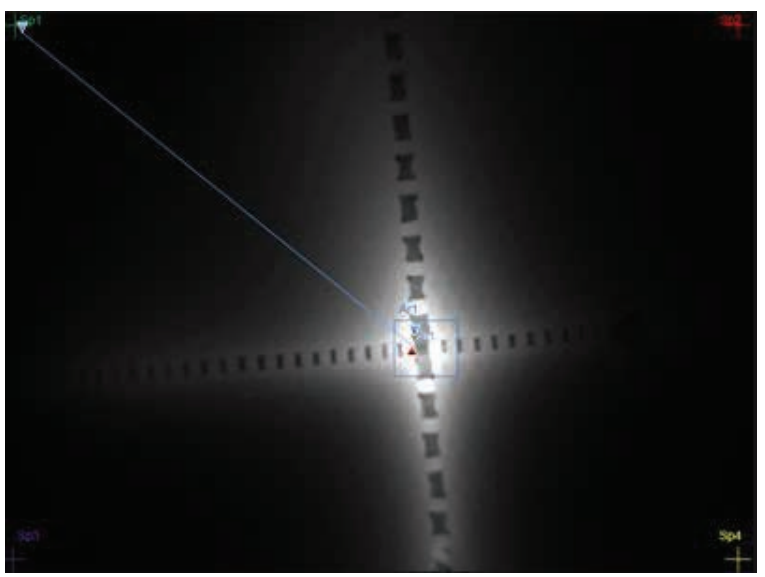

Figure 6. Thermographic image of the heat generated in the contact point when a current flows through it.

\section{Results and discussion}

\section{Contact resistance}

The four electrode-four contact method adapted from the study by Banaszczyk et al [10] was used, with additional steps in the variation of the load and the current.

In the setup of the hanging yarns, the applied current was changed in the following steps: 0.05-0.1-0.2-0.3-0.2-0.10.3-0.5-0.3 A. This stepwise building up and down of the current enables detecting hysteresis. The $\mathrm{V}-\mathrm{I}$ graphs for the three applied weights on the yarns $(2,10$, and $100 \mathrm{~g})$ are shown in Figures 7-9.

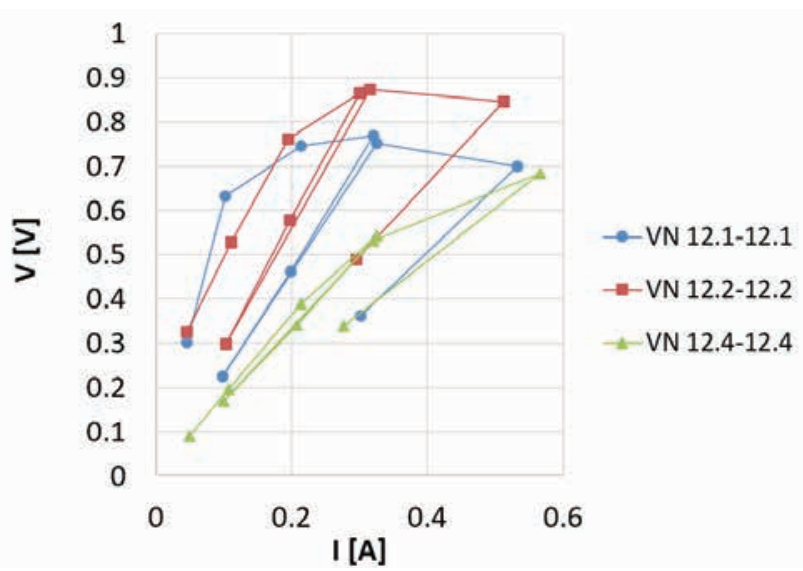

Figure 7. Four-point measurement of two hanging stainless steel yarns using different weights $-2 \mathrm{~g}$.

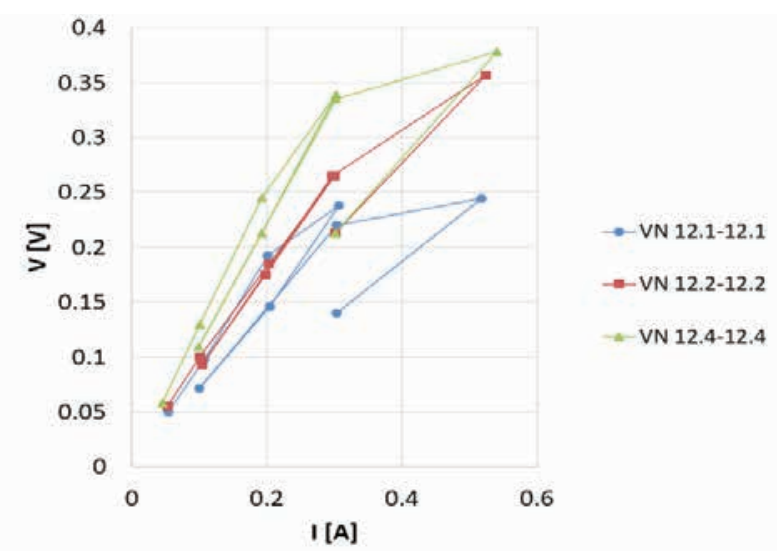

Figure 8. Four-point measurement of two hanging stainless steel yarns using different weights $-10 \mathrm{~g}$.

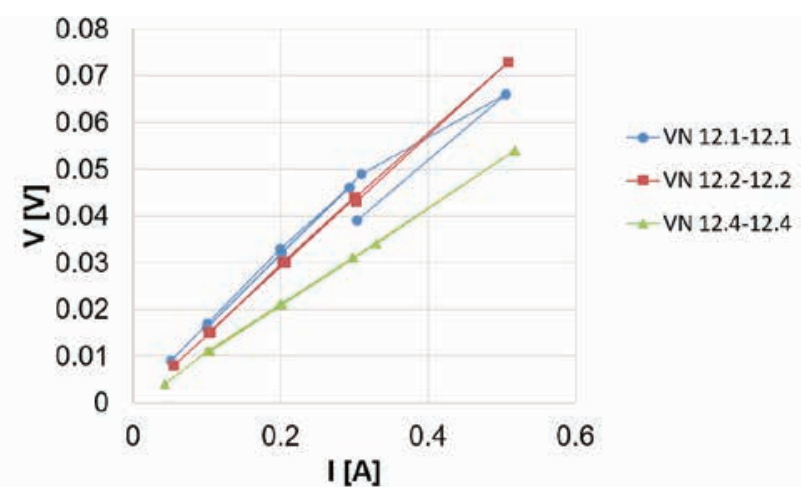

Figure 9. Four-point measurement of two hanging stainless steel yarns using different weights $-100 \mathrm{~g}$.

In most of the situations, especially for the lower weights, a hysteresis effect is observed. Furthermore, following conclusions can be made:

The larger the weight hung on the yarn, the lower the resistance in the contact point.

The trend suggests that the thicker the yarn (i.e. VN12.4), the lower the contact resistance (Figure 10).

At a certain point, the drapeability of yarn is low, creating a smaller contact area than expected. 


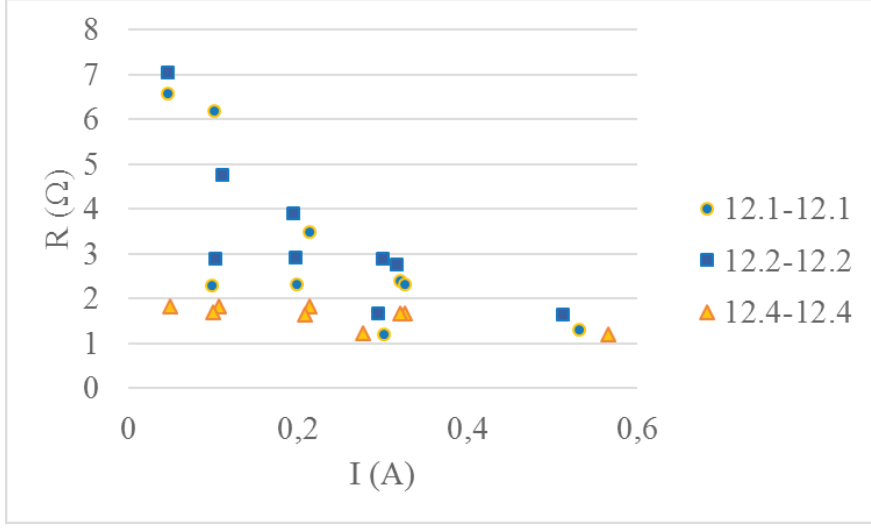

Figure 10. Resistance of three combinations of yarn with $2 \mathrm{~g}$.

The first two conclusions were as expected because in both of the cases there is tighter contact between the two EC yarns and higher contact area, respectively, making it easier for current to flow from one yarn to the other.

Another more remarkable observation is that:

Because the voltage does not increase linearly with increasing current in all cases (especially with lower weights), the contact point of the two interlacing EC yarns does not react as an ohmic resistance.

The $V-I$ graph as shown in Figure 7 has the characteristic of a non-ohmic device. Further investigation showed that the contact has a similar behavior to an MOV.

\section{Varistor}

A varistor is an electrical component with a characteristic $\mathrm{V}-\mathrm{I}$ curve of a non-ohmic conductor (Figure 11). It is a variable resistor, thus a voltage-dependent resistor. Typical for a varistor is that the $\mathrm{V}-\mathrm{I}$ curve is symmetric around the origin. Often varistors are implemented in parallel with a delicate electronic appliance to protect it from power surges. When an overvoltage occurs, the resistance of the varistor in parallel is low and the current will flow to the parallel circuit, grounding it.

An MOV is a specific type of varistor based on metal oxides. In many cases, this metal is $\mathrm{ZnO}$ in combination with oxides from cobalt, manganese, and bismuth [6]. The MOV behavior is a result of the grain boundary. The varistor effect is a result of the reduced electrical conductivity in the boundary of the 10 till $100 \mathrm{~mm}$-sized grains. In the case of an EC contact between

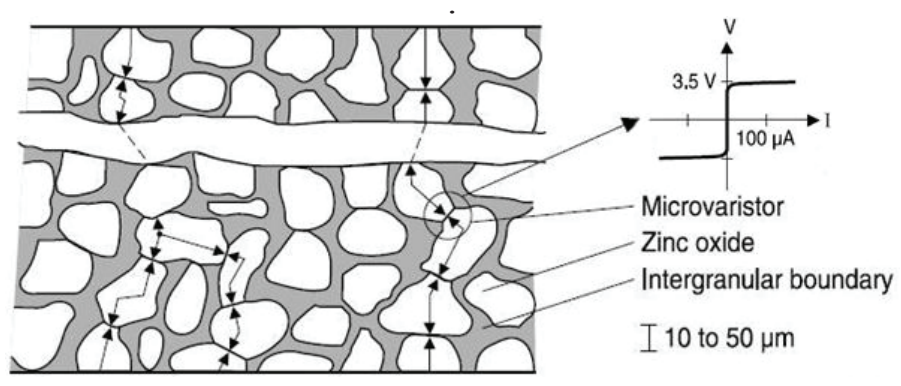

Figure 11. Schematic explaining a commercial metal oxide varistor based on $\mathrm{ZnO}$ [11]. two stainless steel yarns, the grain is the well conductive bulk stainless steel of the monofilaments $\left(\rho=7.4^{\prime} 10^{-7} \mathrm{Wm}\right)$ with a thickness of 12 or $14 \mathrm{Wm}$. The boundary is the more resisting Cr2O3 layer in between $\left(\rho=4^{\prime} 10^{-5} \mathrm{Wm}\right)$. [12].

As a result of quantum tunneling, the voltage over the MOV equals $C \cdot \beta$ in the normal operating region of the MOV ( $C$ and $\beta$ are MOV parameters). In the leakage region (very small currents) the MOV acts ohmic, resulting in a large voltage drop.

Thus, the basic equation characterizing an MOV in its normal operating window is as follows:

$$
V=C \cdot I^{\mathrm{b}}
$$

Where $C$ is the voltage over the MOV at $1 \mathrm{~A}$ and $\beta$ is the slope of the curve of $\log V$ as a function of $\log I$ (Figure 12).

In graphs, the proposed function of the MOV behavior does not apply in the leakage region $(I \leq 0.07 \mathrm{~A})$. Here, the model predicting the resistance and the temperature difference of the EC contact can only be used quantitatively.

Based on the theory of the MOV,

$$
\begin{aligned}
& R=\frac{V}{I} \\
& \log R=\log V-\log I \\
& \log R=\log C+(\beta-1) \log I
\end{aligned}
$$

where $C$ and $\beta$ are MOV parameters.

A simple four electrode-four contact method as shown in Figure 5 on the yarns in the hybrid fabric is enough to determine $C$ and $\beta$. Therefore, current is sent through the EC contact points, and the voltage is recorded. The resulting resistance is calculated and the $\log R-\log I$ graph is plotted. As foreseen by the characteristic of an MOV, the relation between $\log R$ and $\log I$ is linear, with $\beta-1$ being the slope of the line, and log $C$ the intercept.

The MOV parameters for the three contacts can be derived from these plots and are given in Table 2.

At this point, the behavior of the contact resistance in the EC point is clear and modeled. However, the aim was to determine

Table 2. Metal oxide varistor parameters for different combinations of electro-conductive yarns.

\begin{tabular}{|c|c|c|c|}
\hline & $\begin{array}{c}\text { VN12.2- } \\
\text { VN12.2 }\end{array}$ & $\begin{array}{c}\text { VN12.4- } \\
\text { VN12.3 }\end{array}$ & $\begin{array}{c}\text { VN12.4- } \\
\text { VN12.4 }\end{array}$ \\
\hline$b$ & 0.185 & 0.810 & 0.955 \\
\hline $\log C$ & -0.2127 & -0.3967 & -0.4737 \\
\hline$C$ & 0.613 & 2.49 & 0.336 \\
\hline
\end{tabular}




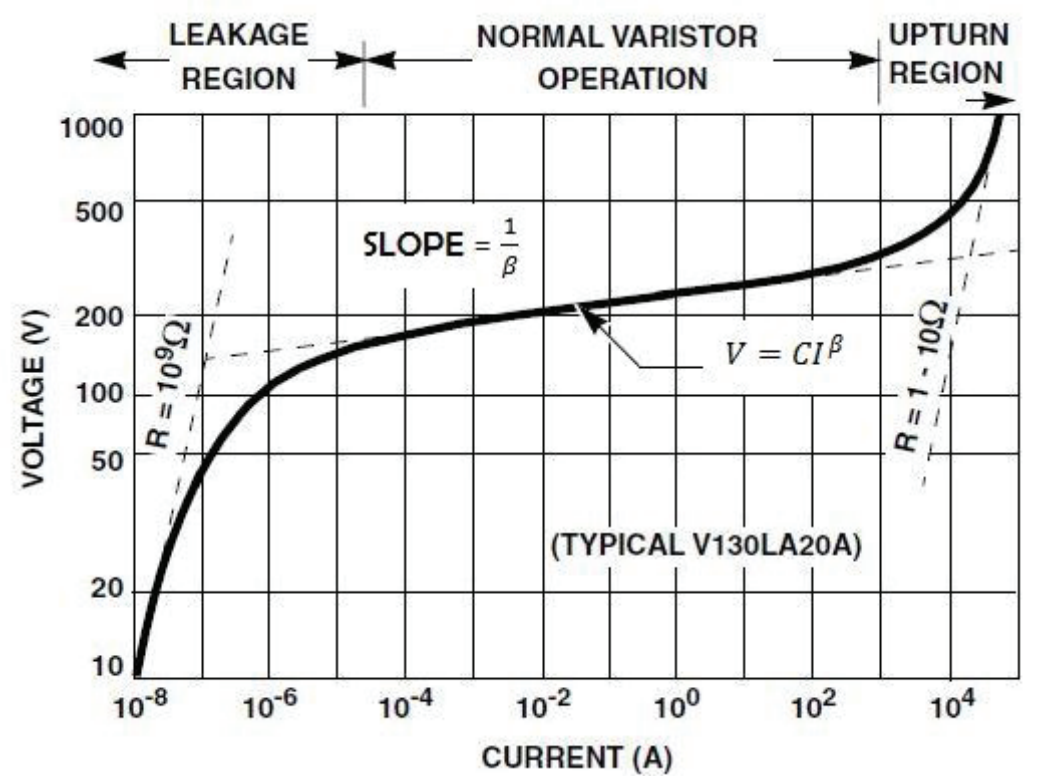

Figure 12. Typical log V-log I curve of a commercial varistor [11].

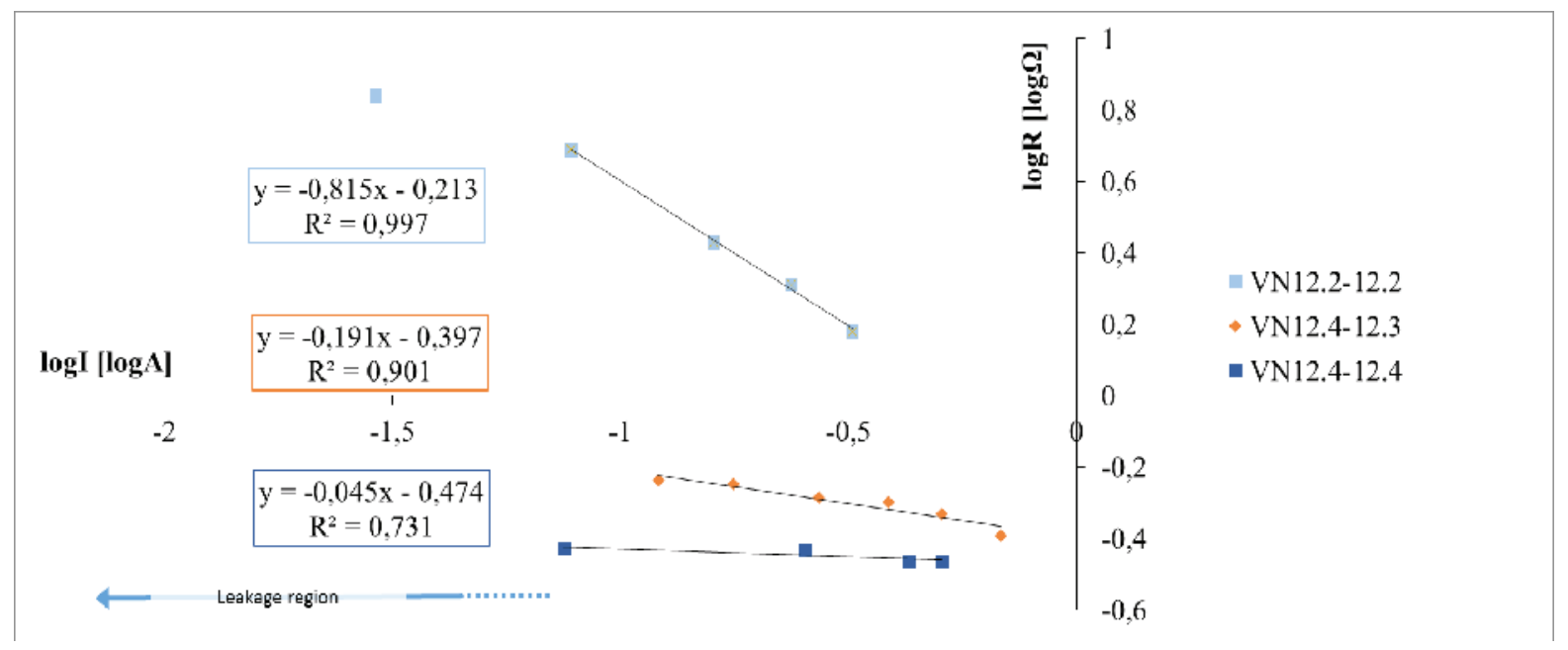

Figure 13. Results of four electrode-four contact measurement. In the leakage region the relation is not according to $V=C \cdot l^{\beta}$.

the relation between the power and the heat generated in the point, which is studied next.

\section{Heating}

The heating of the EC contact, as a result of current flowing through it, is explained by the basic laws of heat transfer [13]. In a steady-state system, the generated heat equals the dissipated heat. The generated heat is the power dissipated in the EC contact $(P=V I)$, and the dissipated heat is the heat transfer by convection to the air.

$$
\begin{aligned}
& Q_{\text {in }}=Q_{\text {diss }}=P=V I \\
& P=h A\left(T-T_{0}\right)=\alpha\left(T-T_{0}\right) \\
& \Delta T=\frac{1}{\alpha} P
\end{aligned}
$$

Where $Q_{\text {in }}$ is the generated heat [W]; $Q_{\text {diss }}$ is the dissipated heat [W]; $P$ is the power dissipated in the EC contact [W]; $h$ is the heat transfer coefficient $\left[\mathrm{W} / \mathrm{m}^{2} \mathrm{~K}\right] ; A$ is area $\left[\mathrm{m}^{2}\right] ; \alpha$ is the heat dissipation coefficient [W/K]; and $T-T_{0}$ is the difference in temperature between the contact and the air [K or $\left.{ }^{\circ} \mathrm{C}\right]$.

Equation (2) means that, according to the basic laws of heat transfer, the temperature rise is proportional to the power dissipated in the EC contact. In addition, $\alpha$ is not dependent on the EC contact, nor on the EC yarns, but on the base fabric and on heat-transfer coefficient. In the same base fabric, $\alpha$ will be independent of the EC yarns if the thermal conductivity through the fabric is uniform. Also, $\Delta T-P$ graphs of different yarns in the same base fabric will be similar. This can be seen in the $\Delta T-P$ graphs in Figure 14, where two groups of EC contacts are marked: a group with VN12.2 as warp yarn (in orange) and a group with VN12.4 as warp yarn (in blue). The slope of the straight lines is proportional to $1 / a$.

There are two curves that clearly diverge from all the others: the combination of yarns VN12.2-VN12.1 and VN12.4-VN12.1. In both cases, the thinnest EC yarn VN12.1 is used in weft direction. Because the weft density is low ( 8 yarns $/ \mathrm{cm})$, the EC yarns and the adjacent polyester yarns make weak contact, 
which results in a lower thermal conductivity. Thus, the heat dissipation coefficient reduces and 1/a increases.

However, if the thermal conductivity of the base fabric changes, $\alpha$ will change too. One of those examples are thin yarns in a loose fabric that highly reduces the heat dissipation coefficient and increases $1 / \alpha$.

It can be concluded that if different EC yarns are in similar contact with the surrounding yarns of the same base fabric, the effect of power on the relative temperature is only determined by the thermal conductivity of the base material.

\section{Mathematical model}

When Equations (1) and (2) are combined, this results in

$$
\begin{aligned}
& \Delta T=\frac{1}{\alpha} P=\frac{1}{\alpha} V I \\
& \Delta T=\frac{1}{\alpha} C I^{\beta+1}
\end{aligned}
$$

This formula is the mathematical model that can predict the temperature difference of an EC contact as a function of the current flowing through it in the normal operating region of the MOV. Three parameters are needed to characterize the EC contact as follows: $\alpha, \beta$ and $C$.

Parameter $\alpha$ is only dependent on the base fabric (i.e. polyester in our case). This makes it unnecessary to make a thermographic measurement of every EC contact. A simple four electrode-four contact measurement determines $\beta$ and C. Hence, a lot of EC contacts with complex exponential graphs are identified with only a handful of thermographic measurements and a simple setup with the four electrode-four contact method.

For our EC yarns, from the graphs of Figure $13, C$ and $\beta$ can be determined for the three-yarn combinations. Then, Figure 15 shows three relations between $\Delta T$ and $I$, two of them are fitted curves to the measured values, and the third one is predicted using the mathematical model. The dotted line (EC contact VN12.4-VN12.3) is based on the assumption that identical fabrics have identical $\alpha$ 's. The points are the measured values of the EC contact and show that there is a good agreement between the model and the measurements, using $\beta$ and $C$ from Figure 13 and $\alpha=0.0021$ (Figure 14).

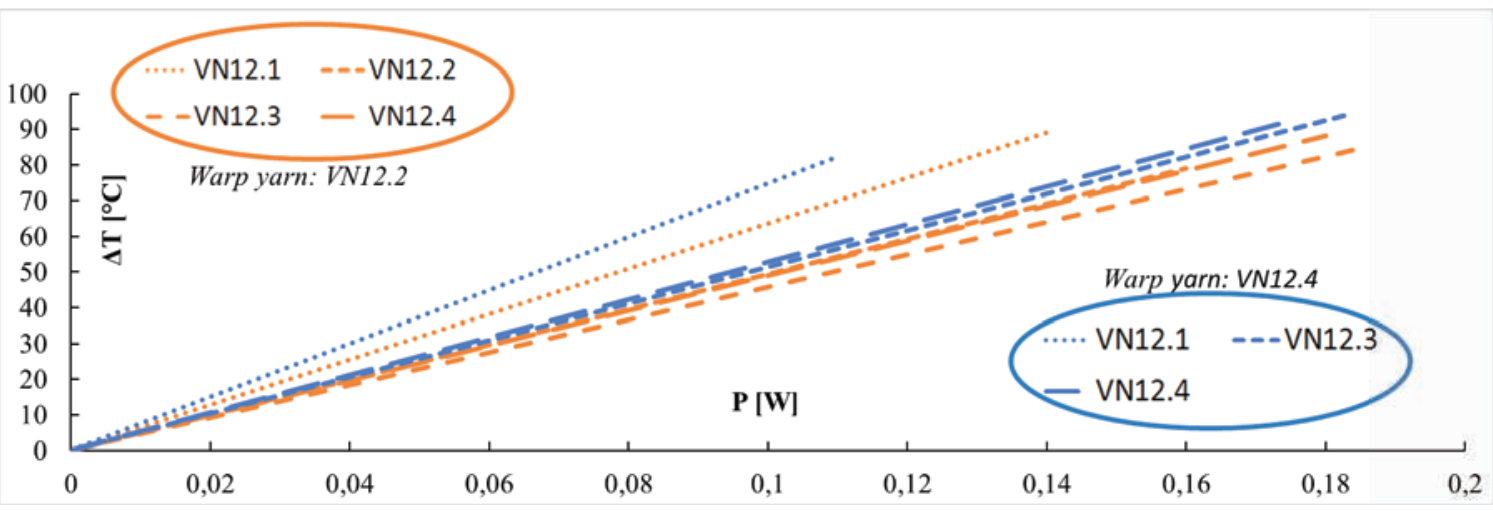

Figure 14. Similar $\Delta T-P$ curves of different electro-conductive $(E C)$ contacts of same plain woven fabrics. Two curves diverge because the EC yarns are thinner and conduction to the adjacent insulating yarns is lower. $a » 0.0021$ and all $R^{2}>0.98$.

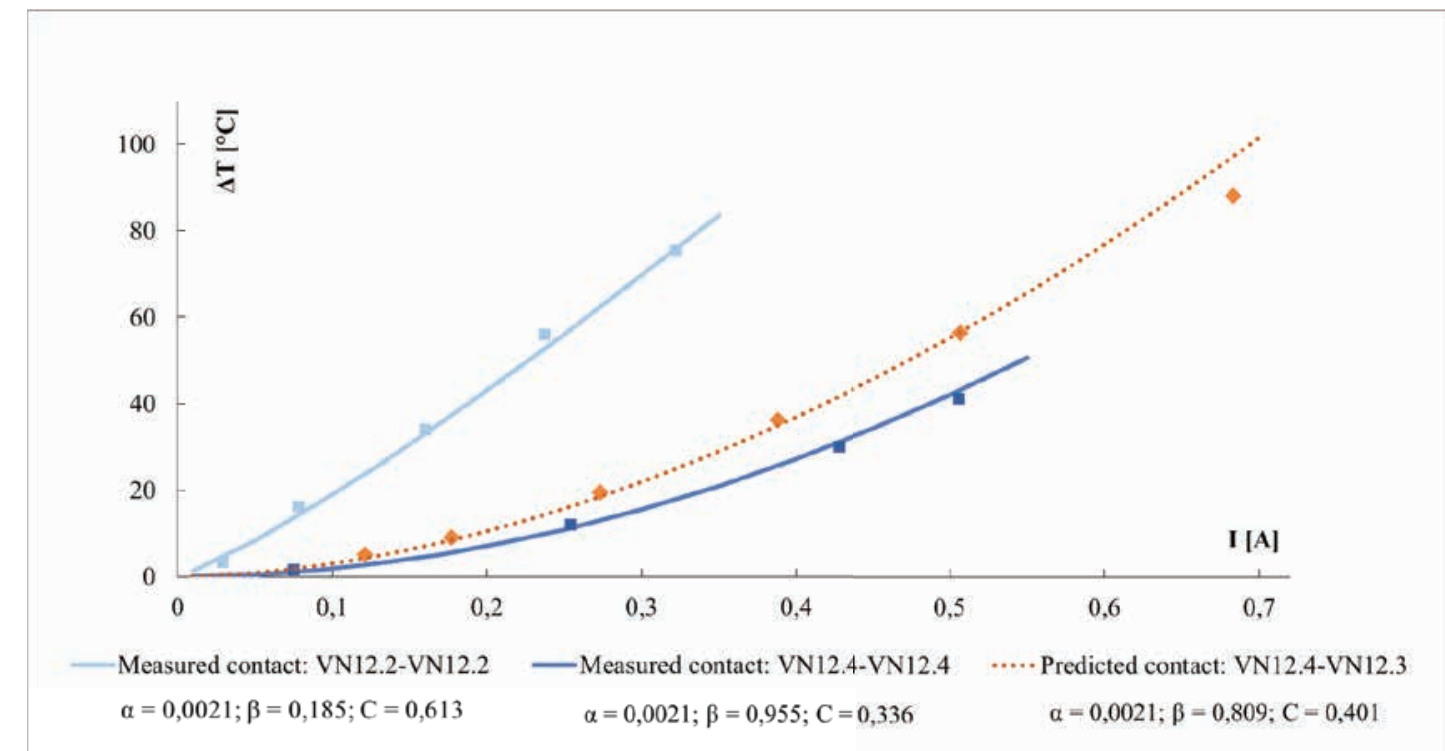

Figure 15. Graphs confirm that there is a good agreement between the mathematical model and the measured data for the combination VN12.4VN12.3. 


\section{Conclusions}

When EC contacts in smart textiles in contact with the skin heat above a temperature of $60^{\circ} \mathrm{C}$ this can cause burn injuries. It is hence important to predict the heat generated in such an EC contact. Studying the EC contacts in hybrid woven fabrics resulted in a mathematical model that can predict the temperature difference of EC contacts as a function of the current flowing through these contacts. In this study, the hybrid fabrics were plain woven with 1,100 dtex polyester yarn as base fabric and four types of EC stainless steel yarn. It was concluded that the EC contacts behave like an MOV and that three parameters are needed to characterize this EC contact: $\alpha, \beta$, and $C$. Parameter $\alpha$ is only dependent on the base fabric (thus the non-conductive material in the fabric) making thermographical measurements unnecessary for every EC contact. A four electrode-four contact measurement of some of the EC contacts in the hybrid woven fabric determines $\beta$ and $C$. Hence, EC contacts with complex exponential graphs are identified with only a handful of thermographic measurements to determine $\alpha$ and a simple setup with the four electrode-four contact method to determine $\beta$ and $C$ of the EC yarns under consideration. The limitation of this study consists of the limited number of EC yarns studied and the model should also be validated for other stainless steel filament yarns with different diameters integrated in woven fabrics other than polyester.

\section{Acknowledgments}

Vlaio (Flanders Agency for Innovation and Entrepreneurship) is acknowledged for its financial support for the WINTEX project (IWT TETRA 130219), in which framework this research was performed.

\section{References}

[1] Dhawan, A., Seyam, A. M., Ghosh, T. K., Muth, J. F. (2004). Woven fabric-based electrical circuits. Part l: evaluating interconnect methods. Textile Research Journal, 913-919.

[2] Stoppa, M., Chiolerio, A. (2014). Wearable electronics and smart textiles: a critical review. Sensors, 11957-11992.
[3] Linz, T., Simon, E., Walter, H. (2012). Modeling embroidered contacts for electronics in textiles. Journal of the Textile Institute, 644-653.

[4] Vasile, S., Deruck, F., Hertleer, C., De Raeve, A., Ellegiers, T., De Mey, G. (2017). Study of the contact resistance of interlaced stainless steel yarns embedded in hybrid woven fabrics. AUTEX Research Journal, 170-176.

[5] Liu, S., Tong, J., Yang, C., Li, L. (2016). Smart e-textile: resistance properties of conductive knitted fabric - Single pique. Textile Research Journal, 1669-1684.

[6] John (2011). http://www.circuitstoday. com/?s=metal+oxide+varistor. [Online]. Website: www. circuitstoday.com.

[7] Moritz, A. R., Henriques, F. C. (1947). Studies of thermal injury II: The relative importance of time and surface temperature in the causation of cutaneous burns. The American Journal of Pathology, 23(5), 695-700.

[8] Bekaert [Online]. Website: https://www.bekaert.com/en/ products/basic-materials/textile/conductive-fibers-andyarns-for-smart-textiles. [Accessed August 2017].

[9] Fluke [Online]. Website: http://en-us.fluke.com/products/ digital-multimeters/fluke-87v-digital-multimeter.html. [Accessed August 2017].

[10] Banaszczyk, J., De Mey, G., Anca, A., Schwarz, A., Van Langenhove, L. (2009). Contact resistance investigation between stainless steel electroconductive yarns, in 16th International Conference: Mixed Design of Integratioed Circuits and Systems, Lodz.

[11] Littlefuse (1999). Website: www.littelfuse.com/data/en/.../ Littelfuse_app-note_an9767.pdf. [Online]. Available: www. littlefuse.com.

[12] EPCOS. (2012). Introduction to Metal Oxide Varistors. [Online]. Website: http://www.powerguru.org/introductioto-metal-oxide- varistors/.

[13] Welty, Wicks, Wilson and Rorrer, Fundamentals of Momentum, Heat and Mass Transfer, 5th Edition ed., Wiley, 2008.

[14] CEN. (2011). CEN/TR 16298 Textiles and textile products - smart textiles - definitions, categorizations, applications and standardization needs. 